\title{
Symptoms of depression are associated with physical inactivity but not modified by gender or the presence of a cardiovascular disease; a cross-sectional study
}

\author{
Retze Achttien ${ }^{1 *}$ (D) Jan van Lieshout ${ }^{1}$, Michel Wensing ${ }^{1,2}$, Maria Nijhuis van der Sanden ${ }^{1}$ and J. Bart Staal ${ }^{1,3}$
}

\begin{abstract}
Background: Depressive symptomatology may act as a barrier to enhance physical activity. This phenomenon is predominantly found in patients with an established cardiovascular disease (CVD) and in female patients. This crosssectional study investigated (1) the association between symptoms of depression and physical inactivity, and (2) whether this association is different between primary and secondary prevention patients, and between men and women.

Methods: The study design concerns a secondary analysis of baseline data from a randomized clinical trial, including primary and secondary prevention patients $(n=2184$; mean age $71.6 \pm 8.94)$, from 34 general practitioner panels. The Rapid Assessment of Physical Activity questionnaire (RAPA) was used to measure patient reported activity levels. Symptoms of depression were determined using the Patient Health Questionnaire (PHQ-9). Multilevel linear regression analysis was used to explore the association between symptoms of depression and physical activity while adjusting for confounders. Gender and whether or not having a CVD were considered as potential effect modifiers for the association between symptoms of depression and inactivity.

Results: Symptoms of depression were associated with lower levels of physical activity. This association was neither different for men and women nor for primary and secondary prevention patients.

Conclusion: In primary care patients' symptoms of depression were associated with physical inactivity. This association was not modified by gender or the presence of a CVD. Future research should focus on lifestyle interventions aiming at the increase of physical activity levels, while emphasizing on improving symptoms of depression in men, women, and patients both with and without a history of CVD.
\end{abstract}

Keywords: Physical activity, Symptoms of depression, CVRM

\section{Background}

Regular physical activity reduces the progression of atherosclerosis, and consequently optimizes the cardiovascular risk profile, and thereby decreases the incidence of cardiovascular events [1]. Exercise capacity is mentioned as one of the strongest modifiable predictors of mortality among patients at risk for developing a cardiovascular disease (CVD) [2]. Despite this well-documented effect, exercise activity is limited among many patients at high

\footnotetext{
* Correspondence: Retze.Achttien@radboudumc.nl

${ }^{1}$ Radboud Institute for Health Sciences, IQ healthcare, Radboud university medical center, Geert Grooteplein 21,6500, HB, Nijmegen, The Netherlands Full list of author information is available at the end of the article
}

cardiovascular risk [3, 4]. Stimulating physical activity is therefore a core component of cardiovascular risk management (CVRM) in primary care [5]. However, to tailor CVRM interventions to individual patients' needs it is critical to identify patient characteristics that are associated with physical inactivity. Patient characteristics such as depressive symptomatology $[6-8]$, female gender $[9,10]$, and suffering a CVD [11] have been found to deteriorate physical activity levels.

Depressive symptomatology may impede the successful stimulation of physical activity and patients' efforts to improve lifestyle in CVD [6-8]. On the other hand, symptoms of depression can be reduced by increasing

(c) The Author(s). 2019 Open Access This article is distributed under the terms of the Creative Commons Attribution 4.0 International License (http://creativecommons.org/licenses/by/4.0/), which permits unrestricted use, distribution, and 
physical activity levels among CVD patients [12-14]. Depressive symptomatology predicts the incidence of CVD, worsens its prognosis, and is mentioned as an independent risk factor for mortality in CVD patients [15-17]. The prevalence of depressive symptomatology increases with age $[11,18]$, smoking status $[18,19]$, and is more prevalent in women compared to men $[9,10]$. Female gender is, independent from experiencing symptoms of depression, also associated with lower activity levels [20, 21]. This might, for instance, be caused by lower perceived self-efficacy and self-management levels in women [22]. Additionally, women experience a CVD at an older age than men, and consequently suffer comorbidity more frequently, resulting in lower activity levels [23-25]. More attention for female gender during CVRM in clinical practice is therefore advocated in the literature $[9,10]$.

Symptoms of depression and consequently lower activity levels are also more frequently reported after a cardiovascular event [20, 26, 27]. Research demonstrating this relationship between depression and inactivity is predominantly conducted during or after (cardiac) rehabilitation in patients with an established CVD (secondary prevention patients) [20]. It may be assumed that this relationship also exists in primary prevention patients (patients at high cardiovascular risk without an established CVD), but data to support this hypothesis are lacking. Yet, insight into possible differences in the association between symptoms of depression and physical inactivity among primary and secondary prevention population might be important for tailoring CVRM to these respective populations [28].

To further explore the association between symptoms of depression and physical inactivity we used baseline data from a large clustered randomized clinical trial (RCT) conducted in primary care patients in the Netherlands [28]. The present study aims to investigate (1) the association between symptoms of depression and physical inactivity, and (2) whether this association is different between primary and secondary prevention patients, and between men and women. These analyses should contribute to our understanding about how to tailor patient-specific lifestyle interventions aiming at a healthy and physically active lifestyle, offered during CVRM in primary care.

\section{Methods}

\section{Study design}

The study design concerns a cross-sectional secondary analysis of baseline data from a clustered RCT [28]. The RCT was part of the international Tailored Implementation for Chronic Diseases (TICD) project. This project developed and tested programs to improve healthcare for patients with chronic diseases in five European countries, and examined methods for tailoring such programs to target groups and settings [29]. The Medical Ethics committee Arnhem-Nijmegen, the Netherlands, waived approval (number 2013/229) for the RCT [30]. The declaration of Helsinki was followed. In our study, we followed the STrengthening the Reporting of OBservational studies in Epidemiology (STROBE) statement to report our cross-sectional study [31].

\section{Setting}

A random sample of 1600 general practices in seven geographical areas in the Netherlands was invited to participate in the RCT [28], resulting in a sample of 34 practices and 2184 patients that were included in our study. Baseline data, used in our study, were collected by the general practitioner (GP) or a nurse practitioner in the years 2013 and 2014. GP practices were only invited to participate if a practice assistant was working in the practice with CVRM in the portfolio.

\section{Participants}

All patients were 18 years or older and were able to provide informed consent. Exclusion criteria were diabetes mellitus, pregnancy and lactation, terminal illness, cognitive impairment, and insufficient mastery of the Dutch language hindering reading and answering the questionnaires. We included the baseline data from a RCT [28] that focussed on CVRM for primary and secondary prevention patients. Primary prevention patients had an estimated 10 -year risk score of $20 \%$ or higher for morbidity and mortality due to CVD. Secondary prevention patients had a cardiovascular event (predominantly coronary artery disease or less frequently a stroke) in history or suffered from a chronic heart disease or peripheral arterial disease. Patient selection was based on the following International Classification of Primary Care (ICPC) codes: K74-K76, K85-K92, K99.1, and T93.

\section{Variables}

The outcome variable was the degree of physical activity measured by the patient reported Rapid Assessment of Physical Activity questionnaire (RAPA) [32]. The predictor variable was symptoms of depression measured by the Patient Health Questionnaire-9 (PHQ-9) [33]. Gender and whether or not suffering a CVD were considered potential effect modifiers for the association between symptoms of depression and inactivity. The experienced degree of control on own health status, measured using the Patient Activation Measure questionnaire (PAM) [34], age, and smoking status were considered as potential confounders.

\section{Data sources and measurements}

Data were collected using structured questionnaires. Questionnaires were sent by mail to all patients and 
returned by mail as well. Data collection was performed equally in primary and secondary prevention patients.

\section{Study size}

The sample size that was used in the RCT [28] on which we focused was based on a sample size calculation for the cluster randomized trial [30]. The calculation indicated that 450 patients per group (primary or secondary prevention) would be needed for the RCT (15 primary and 15 secondary prevention patients per cluster, sampled in 30 practices). The patient response to participate in the RCT was $41.8 \%$ of the invited patients at baseline [28].

\section{Quantitative variables}

All patients filled out the RAPA, PHQ-9, PAM and a smoking questionnaire. The RAPA (9-items) is a valid measure of physical activity for use in clinical practice [32]. RAPA evaluates a wide range of physical activity levels, from sedentary to vigorous activity, as well as strength and flexibility training [35]. Each question has a 'Yes' or 'No' option. The score ranges from 1 to 7 ; a score of $6-7$ points is considered "active", 4-5 points as "suboptimal active", and $\leq 3$ points is defined as "sedentary" to "under-active regular-light activities". Missing values were only considered as such if no questions were filled in. In the data-analysis, we used the RAPA as a continuous score.

The PHQ-9 is a validated depression module for primary care, which scores each of the nine Diagnostic and Statistical Manual of Mental Disorders (DSM-IV) criteria as "0" (not at all) to " 3 " (nearly every day), with a total score ranging from 0 to 27 points [32]. The total score can also be categorized in 5 severity levels; $0-4$ none, 5-9 mild, 10-14 moderate, 15-19 moderately severe, and 20-27 severe symptoms of depression. The total score on the PHQ-9 was used as a continuous value in the statistical analysis.

The PAM is a valid and reliable instrument assessing patients' activation measure [34]. The PAM survey measures patients on a 13-52 scale, revealing insight into an array of health-related characteristics (attitudes, motivators, behaviors, and outcomes). In case 1 or 2 separate questions were not answered (missing), the total PAM score was subsequently divided by 12 or 13 and multiplied by 13. The PAM was excluded from analysis if fewer than 11 out of 13 questions were answered. This strategy was in accordance with the guideline for interpretation of the questionnaire [34].

The smoking status was derived from the first question of a standardized questionnaire on smoking habits [36]. There were 4 possible answers options: "Yes I smoke", "No I quite last 6 months", "No I quite more than 6 months ago", or "No I never smoked". We dichotomized this into Yes or No in the analysis. The covariate age was used as a continuous variable in the analysis.

\section{Statistical methods}

Data analysis was performed using SPSS (version 23, IBM Corp.) Data were described using means, standard deviations, numbers, and percentages. Patient characteristics and the outcome of (completed) questionnaires were presented for the total study population. Outcomes were presented on a dichotomous or continuous scale.

First, independent sample T-tests and/ or Chi-square tests were used to compare the outcome variable, the predictor, and potential confounders between primary and secondary prevention patients, and between male and female patients. Second, the assumptions for multilevel linear regression analyses (i.e. linear relationship between the independent and dependent variables, multivariate normality, no or little multicollinearity, and homoscedasticity) were checked [37]. Then, multilevel linear regression analysis (95\% confidence intervals (CIs)), using random slopes and intercepts, was performed to determine the association between symptoms of depression and physical inactivity. We adjusted for the potential confounders; score on the PAM questionnaire, age and smoking, gender and whether or not suffering a CVD. Third, we explored if the association between symptoms of depression and physical inactivity was different between primary and secondary prevention patients, and between men and women, by adding the interaction terms (primary/ secondary prevention group*PHQ-9 and gender"PHQ-9 respectively) to separate models. A $p$ value $<0.05$ was considered as statistically significant.

\section{Results}

\section{Participants and descriptive data}

Table 1 presents the descriptive statistics and responses to the questionnaires.

In total, 2184 patients were included in our study (1250 primary and 934 secondary prevention patients), consisting of 761 female patients and 1423 male patient. The high-risk population consisted of 33\% (mean age $75.7 \pm 7.0)$ female patients and in the CVD population $38 \%$ (mean age $68.8 \pm 13.1$ ). Primary prevention patients were significantly older than secondary prevention patients $(p<0.05 ; 73.99 \pm 6.99$ vs $68.50 \pm 11.73)$. In total, $110(5 \%)$ patients filled out the RAPA inadequately. For the PHQ-9, PAM and smoking status, the non-response was as follows: $76(3,4 \%), 68(3,1 \%)$, and $57(2,6 \%)$.

\section{Outcome data}

Female patients in the primary prevention group scored on average $4.40( \pm 1.62)$ points on the RAPA and in the secondary prevention group $4.61( \pm 1.55)$ points. This difference is statistically significant $(p<0.05)$. Male patients were in both groups physically more active $(p<0.01)$ compared to female patients (primary prevention group $5.01 \pm 1.54$ vs $4.4 \pm 1.62$ and secondary prevention 
Table 1 Descriptive characteristics of the study population

\begin{tabular}{|c|c|c|c|c|c|c|}
\hline & \multicolumn{6}{|l|}{ Patient group } \\
\hline & \multicolumn{3}{|c|}{ Primary prevention patients } & \multicolumn{3}{|c|}{ Secondary prevention patients } \\
\hline & $\begin{array}{l}\text { Female } \\
(n=407)\end{array}$ & $\begin{array}{l}\text { Male } \\
(n=843)\end{array}$ & $\begin{array}{l}\text { Total } \\
(n=1250)\end{array}$ & $\begin{array}{l}\text { Female } \\
(n=354)\end{array}$ & $\begin{array}{l}\text { Male } \\
(n=580)\end{array}$ & $\begin{array}{l}\text { Total } \\
(n=934)\end{array}$ \\
\hline Age in years, mean, SD & $75.74 \pm 7.10$ & $73.15 \pm 6.79$ & $73.99 \pm 6.99$ & $68.83 \pm 13.11$ & $68.31 \pm 10.80$ & $68.50 \pm 11.73$ \\
\hline RAPA, mean, SD & $4.4 \pm 1.62$ & $5.01 \pm 1.54$ & $4.82 \pm 1.59$ & $4.61 \pm 1.55$ & $5.02 \pm 1.58$ & $4.87 \pm 1.58$ \\
\hline Active (RAPA 6-7 points), $n$, (\%) & $151(37.1 \%)$ & $430(51.0 \%)$ & $581(46.5 \%)$ & $137(38.7 \%)$ & $299(51.6 \%)$ & $436(46.7 \%)$ \\
\hline Suboptimal (RAPA 4-5 points), n, (\%) & $97(23.8 \%)$ & $205(24.3 \%)$ & $302(24.2 \%)$ & $97(27.4 \%)$ & $132(22.8 \%)$ & $229(24.5 \%)$ \\
\hline Underactive (RAPA $\leq 3$ points), $n$, (\%) & $129(31.8 \%)$ & $172(20.4 \%)$ & $301(24.1 \%)$ & $98(27.7 \%)$ & $127(21.9 \%)$ & $225(24.1 \%)$ \\
\hline Missing, $n,(\%)$ & $30(7.4 \%)$ & $36(4.3 \%)$ & $66(5.3 \%)$ & $22(6.2 \%)$ & $22(3.8 \%)$ & $44(4.7 \%)$ \\
\hline PHQ-9, mean, SD & $2.67 \pm 3.35$ & $1.78 \pm 3.08$ & $2.05 \pm 3.19$ & $4.23 \pm 4.48$ & $2.57 \pm 3.49$ & $3.18 \pm 3.96$ \\
\hline No symptoms of depression (0-4), $n,(\%)$ & $298(73.2 \%)$ & 719 (85.3\%) & 1017 (81.4\%) & $208(58.8 \%)$ & $445(76.7 \%)$ & $653(69.9 \%)$ \\
\hline Mild symptoms of depression (5-9), $n,(\%)$ & $64(15.7 \%)$ & $77(9.1 \%)$ & $141(11.3 \%)$ & $93(26.3 \%)$ & $84(14.5 \%)$ & $177(19.0 \%)$ \\
\hline Moderate symptoms of depression (10-14), n, (\%) & $13(3.2 \%)$ & $22(2.6 \%)$ & $35(2.8 \%)$ & $26(7.3 \%)$ & $25(4.3 \%)$ & $51(5.5 \%)$ \\
\hline Moderately severe symptoms of depression (15-19), n, (\%) & $7(1.7 \%)$ & $3(0.4 \%)$ & $10(0.8 \%)$ & $10(2.8 \%)$ & $4(0.7 \%)$ & $14(1.5 \%)$ \\
\hline Severe symptoms of depression (20-27), $n,(\%)$ & 0 & $4(0.5 \%)$ & $4(0.3 \%)$ & $4(1.1 \%)$ & $2(0.3 \%)$ & $6(0.6 \%)$ \\
\hline Missing, $n,(\%)$ & $25(6.1 \%)$ & $18(2.1 \%)$ & $43(3.4 \%)$ & $13(3.7 \%)$ & $20(3.4 \%)$ & $33(3.5 \%)$ \\
\hline PAM, mean, SD & $35.31 \pm 8.47$ & $36.70 \pm 8.55$ & $36.27 \pm 8.5$ & $36.41 \pm 7.59$ & $38.45 \pm 7.56$ & $37.68 \pm 7.63$ \\
\hline Missing, n, (\%) & $28(7.4 \%)$ & $12(1.4 \%)$ & $40(3.3 \%)$ & $14(4.1 \%)$ & $14(2.5 \%)$ & $28(3.1 \%)$ \\
\hline \multicolumn{7}{|l|}{ Smoking status } \\
\hline YES, $n,(\%)$ & $23(5.9 \%)$ & 87 (10.5\%) & $91.2(8.8 \%)$ & $52(15.2 \%)$ & 77 (13.6\%) & $129(13.8 \%)$ \\
\hline NO, n, (\%) & 366 (94.1\%) & 471 (89.5\%) & 1107 (89.5\%) & 291 (84.8\%) & $490(86.4 \%)$ & 781 (86.2\%) \\
\hline Missing, $n,(\%)$ & $18(4.4 \%)$ & 15 (1.8\%) & 33 (2.7\%) & $11(3.1 \%)$ & 13 (2.2\%) & $24(2.5 \%)$ \\
\hline
\end{tabular}

RAPA Rapid Assessment of Physical Activity Questionnaire, PHQ-9 Patient Health Questionnaire-9, PAM Patient Activation Measure questionnaire

group $5.02 \pm 1.58$ vs $4.61 \pm 1.55, P<0.01)$. In the primary and secondary prevention group, about $50 \%$ of the patients scored "active" on the RAPA (6-7 points), 25\% scored "less than suboptimal active" (4-5 points), and 25\% scored "sedentary" to "under-active regular-light activities" (1-3 points).

Most patients in this study did not report symptoms of depression $(81.4 \%$ in the primary prevention group and $69.9 \%$ in the secondary prevention group). Mild and moderate symptoms of depression were more frequently reported in the secondary prevention group compared to the primary prevention group (mild symptoms of depression; $11,3 \%$ vs $19.0 \%$ and moderate symptoms of depression $2,8 \%$ vs $5.5 \%, p<0.01$ ). Reported symptoms of depression were significantly higher in patients suffering a CVD (women: $4.23 \pm 4.48$, men $2.57 \pm 3.48, p<0.01$ ) compared to primary prevention patients (women: $2.67 \pm 3.35$, men $1.78 \pm 3.08, p<0.01$ ).

In the secondary prevention group, both female and male patients were more often reported to be a smoker compared to the primary prevention group. The primary prevention group reported lower scores on the PAM compared to secondary prevention patients $(36.27 \pm 8.55$ vs $37.68 \pm 7.63, p<0.01)$. There was no difference in PAM score between women and men.

\section{Main results}

Assumptions for multilevel linear regression analyses were not violated and no effect for clustering was found $(\mathrm{ICC}=0.02)$. Table 2 presents the multilevel linear regression analyses concerning the association between symptoms of depression and physical inactivity (using the RAPA as a continuous variable), adjusted for confounders. There was a significant association $(p<0.01)$ between symptoms of depression and physical inactivity, indicating that a 1-point increase on the PHQ-9 questionnaire is associated with a decrease of 0.06 on the RAPA. The association between symptoms of depression and physical inactivity was neither modified by the interaction term primary/secondary prevention group*PHQ-9 $(p=0.47$ : Table 2 , model 2$)$ nor by the interaction term gender* PHQ-9 ( $p=0.44$ : Table 2 , model 3$)$.

\section{Discussion}

\section{Key results}

The aim of this study was to investigate (1) the association between symptoms of depression and physical inactivity, and (2) if this association is different between primary and secondary prevention patients, and between men and women. Our results revealed that depression was associated with physical inactivity in both primary 
Table 2 Results of multilevel linear regression analyses presenting the association between symptoms of depression and physical activity

\begin{tabular}{|c|c|c|c|c|c|c|c|c|c|c|c|c|}
\hline \multirow[b]{2}{*}{ RAPA (1-7) } & \multicolumn{4}{|c|}{$\begin{array}{l}\text { Model } 1 \\
\text { 95\% C.I. }\end{array}$} & \multicolumn{4}{|c|}{$\begin{array}{l}\text { Model } 2 \\
95 \% \text { C.I. }\end{array}$} & \multicolumn{4}{|c|}{$\begin{array}{l}\text { Model } 3 \\
\text { 95\% C.I. }\end{array}$} \\
\hline & B & Lower & Upper & P & B & Lower & Upper & P & $B$ & Lower & Upper & $P$ \\
\hline PHQ-9 (0-27) & -0.06 & -0.08 & -0.04 & $<0.01$ & -0.08 & -0.14 & -0.02 & 0.01 & -0.09 & -0.15 & -0.02 & 0.01 \\
\hline Gender (M/ F) & 0.33 & 0.18 & 0.48 & $<0.01$ & 0.33 & 0.18 & 0.48 & $<0.01$ & 0.29 & 0.10 & 0.47 & $<0.01$ \\
\hline Patient group (SP/PP) & -0.01 & -0.15 & 0.14 & 0.92 & -0.05 & -0.22 & 0.12 & 0.55 & -0.02 & -0.16 & 0.12 & 0.82 \\
\hline Age $(Y)$ & -0.03 & -0.03 & -0.02 & $<0.01$ & -0.03 & -0.04 & -0.02 & $<0.01$ & -0.03 & -0.04 & -0.02 & $<0.01$ \\
\hline Smoking status (Y/ N) & 0.35 & 0.13 & 0.57 & $<0.01$ & 0.35 & 0.13 & 0.56 & $<0.01$ & 0.34 & 0.13 & 0.56 & $<0.01$ \\
\hline PAM (0-13) & 0.02 & 0.01 & 0.03 & $<0.01$ & 0.02 & 0.01 & 0.03 & $<0.01$ & 0.02 & 0.01 & 0.03 & $<0.01$ \\
\hline SP/PP group*PHQ-9 & & & & & 0.01 & -0.02 & 0.05 & 0.47 & & & & \\
\hline Gender*PHQ-9 & & & & & & & & & 0.02 & -0.02 & 0.05 & 0.44 \\
\hline
\end{tabular}

* means SP/ PP group multiplied by PHQ-9

Rapid Assessment of Physical Activity Questionnaire. PHQ9 Patient Health Questionnaire 9, PAM Patient Activation Measure questionnaire score, SP secondary prevention patients, $P P$ primary prevention patients

and secondary prevention patients in primary care. However, this association was not modified by gender or the presence of a CVD.

\section{Strengths and limitations}

Patient baseline data (from the RCT [28]) were collected from GP databases, considering an even distribution of primary and secondary prevention patients. However, selection bias may have influenced the outcome in our study. In total 34 GP facilities participated in the RCT, while 1600 practices were invited to participate [28]. In the practices, a nurse practitioner had to be employed who had CVRM in the portfolio. Therefore, it is possible that predominantly data from higher quality GP practices were used in our study.

The selection criteria for patients being included in our study were standardized, using ICPC diagnose codes (mostly objective criteria). Selection bias may have occurred in the RCT by a low patient response $(41.8 \%$ at baseline) to the invitation to participate [28]. It could be, for example, that patients with no or minor depression predominately participated in our study. However, this selection bias was probably the same in primary and secondary prevention group. Other factors (covariates) associated with physical activity levels and/ or symptoms of depression, for which we did not adjust in the analysis, may have been different between the primary and secondary prevention group. Factors such as personal (scheduling, motivation), social (family support) and environmental (cost, access) barriers and biological correlates (body mass index, physical co-morbidities) may have acted as confounders $[6,20,24,38]$. In particular, the body mass index might have been a potential confounder, as it has been reported to be associated with lower activity levels in depressed patients [39]. We did not correct for the BMI variable in our model, due to the substantial number of missing data (77\%). This is a limitation of our study.

Validated questionnaires were used in our study. Only a limited number of patients did not fill out the questionnaires correctly. We consider this as a strength of our study. Due to the cross-sectional study design, however, it is not possible to draw any causal relationship. This is an important limitation of this study design.

\section{Interpretation}

We revealed the association between symptoms of depression and physical inactivity in a primary care population consisting of primary and secondary prevention patients. In addition, to our knowledge, this is the first study that investigates the possible difference in the association between depression and inactivity between primary and secondary prevention patients, and between men and women.

Increased symptoms of depression and consequently physical inactivity were previously reported in secondary prevention patients during or after cardiac rehabilitation $[11,25]$. In addition, it was reported that adults with major depression disorders do have low levels of physical activity [39]. Moreover, in secondary prevention patients it has also been found that inactive patients are more often depressed and that the more patients get depressed, the less active they become $[12,13]$. This is in line with our findings in secondary prevention patients, and we also found that this association exists in primary prevention patients. It should be noted that our population predominantly had no $(81.4 \%$ of the primary and $69.9 \%$ of the secondary prevention patients) or only minor symptoms of depression.

The association between depression and physical inactivity was not always consistently reported. In a narrative review [20] for example, authors concluded that during cardiac rehabilitation, depression was associated 
with physical inactivity in $63 \%$ of the studies included. This association was lower (57\% of the studies included) in secondary prevention patients who did not attend a rehabilitation program [20]. Differences in findings might be due to the level of symptoms of depression that patients suffer and the degree of patients' inactivity. Patients included in our study had relatively low levels of symptoms of depression compared to other studies [20]. In the secondary prevention group, this could be the result of multidisciplinary (cardiac) rehabilitation that patients may have followed prior to entering our study, which aims for improving symptoms of depression.

Overall, our study population was also less active compared to patients included in other research [20,40]. This could be explained by the fact that we included older patients, who might suffer more comorbidity and are therefore less active [23-25]. On the other hand, aging is reported to be inconsistently associated with lower activity levels and exercise adherence among secondary prevention patients [20]. Aging is, however, related to higher prevalence's of symptoms of depression [11, 18]. Attending cardiac rehabilitation in the past seems not to affect activity levels in the long term and might therefore not affect our findings [4].

We reported higher activity levels in men compared to women in both the primary and secondary prevention group. This has also been reported in earlier research $[9$, $10,23,24]$. In our study, men and women scored equally on the PAM, while in previous research it was shown that women had lower self-efficacy than men [22]. In our study we found lower scores on the PAM in the primary prevention group compared to the secondary prevention group. A positive impact of higher self-regulation and self-efficacy, proper health status, positive intention, internal locus of control, and absence of negative illness beliefs is, for example, are consistently related to exercise maintenance in CVD population [20, 41] and the non-disease population [25]. Therefore, we treated the PAM as a potential confounder in the analysis. And finally, in the secondary prevention group were more smokers than in the primary prevention group. Smoking has previously been reported as inconsistently related to inactivity among CVD patients [20,42], but is associated with increased symptoms of depression levels [19]. Smoking was therefore also treated as a potential confounder in the analysis.

\section{Generalizability}

Dutch citizens are registered by a GP. The GP has a medical record of almost all patients, and therefore every patient who is eligible for CVRM within the participating practices is in the original selection from which the sample has been taken. Also, the distribution of one third female and two third male patients is representative of the CVRM population in the western primary healthcare facilities. Only 3.8\% of the patients had no Dutch nationality, and therefore generalizability to people originating from non-western countries could be limited.

\section{Conclusion}

Symptoms of depression are associated with physical inactivity. This association was not modified by gender and the presence of a CVD. Future research should focus on lifestyle interventions aiming at the increase of physical activity levels while simultaneously improving symptoms of depression, despite gender difference and the presence of a CVD. Longitudinal research should be performed to re-confirm the present findings.

\section{Abbreviations}

CVD: Cardiovascular disease; CVRM: Cardiovascular risk management; DSMIV: Diagnostic and statistical manual of mental disorders; GP: General practitioner; ICPC: International classification of primary care; PAM: Patient activation measure questionnaire; PHQ-9: Patient health questionnaire; RAPA: Rapid assessment of physical activity questionnaire; RCT: Clustered randomized clinical trial; STROBE: STrengthening the reporting of OBservational studies in epidemiology; TICD: Tailored implementation for chronic diseases

\section{Acknowledgements}

We are grateful to Dr. G. Irwin for his statistical advice and English editing.

\section{Funding}

None.

Availability of data and materials

The dataset used and/ or analyzed during the current study are available from the corresponding author on reasonable request.

\section{Authors' contributions \\ $R A, M W, J v L, M N v d S$, and JBS contributed substantially to the study concept and research questions. MW and JVL delivered the database. RA, JVL and JBS analyzed and interpreted the data. The statistical analyses and interpretation were discussed with the other authors. RA wrote the first concept of the manuscript. Concept versions of the manuscript were discussed and edited by JVL, JBS, MNvdS, and MW. All authors read, comment and approved the final submitted version of the manuscript. All authors agreed both to be personally accountable for the author's own contributions and ensure that questions related to the accuracy or integrity of any part of the work, even ones in which the author was not personally involved, were appropriately investigated, resolved, and resolute documented in the literature.}

\section{Ethics approval and consent to participate}

Data from a previously conducted Randomized clinical trial (RCT) were used. The Medical Ethics committee Arnhem-Nijmegen, the Netherlands, waived approval (number 2013/229) for this RCT. All subjects gave written informed consent. The declaration of Helsinki was followed.

\section{Consent for publication}

Not applicable.

\section{Competing interests}

The authors declare that they have no competing interest.

\section{Publisher's Note}

Springer Nature remains neutral with regard to jurisdictional claims in published maps and institutional affiliations.

\section{Author details}

${ }^{1}$ Radboud Institute for Health Sciences, IQ healthcare, Radboud university medical center, Geert Grooteplein 21, 6500, HB, Nijmegen, The Netherlands. ${ }^{2}$ Health services research and implementation science in healthcare, 
Heidelberg University, Heidelberg, Germany. ${ }^{3}$ Research group Musculoskeletal Rehabilitation, HAN University of Applied Sciences, Nijmegen, The Netherlands.

Received: 27 April 2018 Accepted: 24 March 2019

Published online: 25 April 2019

\section{References}

1. Scrutinio D, Temporelli PL, Passantino A, Giannuzzi P. Long-term secondary prevention programs after cardiac rehabilitation for the reduction of future cardiovascular events: focus on regular physical activity. Futur Cardiol. 2009; 5:297-314.

2. Myers J, Prakash M, Froelicher V, Do D, Partingstion S, Atwoord JE. Exercise capacity and mortality among men referred for exercise testing. N Engl J Med. 2002;346:793-801.

3. Sunamura M, Ter Hoeve N, van den Berg-Emons HJ, Haverkamp M, Redekop K, Geleijnse ML, et al. OPTImal CArdiac REhabilitation (OPTICARE) following acute coronary syndromes: rationale and design of a randomised, controlled trial to investigate the benefits of expanded educational and behavioural intervention programs. Neth Heart J. 2013;21:324-30.

4. ter Hoeve N, Huisstede BM, Stam HJ, van Domburg RT, Sunamura M, Van den berg-Emons RJ. Does cardiac rehabilitation after an acute cardiac syndrome lead to changes in physical activity habits? Systematic review. Phys Ther. 2015:95:167-79.

5. Kotseva K, Wood D, De Bacquer D, De Backer G, Rydén L, Jennings C, et al. EUROASPIRE IV: a European Society of Cardiology survey on the lifestyle, risk factor and therapeutic management of coronary patients from 24 European countries. Eur J Prev Cardiol. 2016;23:636-48.

6. Cooper AF, Jackson G, Weinman J, Horne R. Factors associated with cardiac rehabilitation attendance: a systematic review of the literature. Clin Rehabil. 2002;16:541-52.

7. Glazer KM, Emery CF, Frid DJ, Banyasz RE. Psychological predictors of adherence and outcomes among patients in cardiac rehabilitation. J Cardpulm Rehabil. 2002;22:40-6.

8. Lichtman JH, Froelicher ES, Blumenthal JA, Carney RM, Doering LV, FrasureSmith $\mathrm{N}$, et al. Depression as a risk factor for poor prognosis among patients with acute coronary syndrome: systematic review and recommendations: a scientific statement from the American Heart Association. Circulation. 2014:129:1350-69.

9. Appelman Y, van Rijn BB, Ten Haaf ME, Boersma E, Peters SA. Sex differences in cardiovascular risk factors and disease prevention. Atherosclerosis. 2015;241:211-8.

10. Moller-Leimkuhler AM. Women with coronary artery disease and depression: a neglected risk group. World J Biol Psychiatry. 2008;9:92-101.

11. Armstrong NM, Meoni LA, Carlson MC, Xue QL, Bandeen-Roche K, Gallo JJ, et al. Cardiovascular risk factors and risk of incident depression throughout adulthood among men: the Johns Hopkins precursors study. J Affect Disord. 2017:214:60-6

12. Hearing CM, Chang WC, Szuhany KL, Deckersbach T, Nierenberg AA, Sylvia LG. Physical exercise for treatment of mood disorders: a critical review. Curr Behav Neurosci Rep. 2016;3:350-9.

13. Teychenne M, Ball K, Salmon J. Physical activity and likelihood of depression in adults: a review. Prev Med. 2008;46:397-411.

14. Schuch FB, Vancampfort D, Firth J, Rosenbaum WFB, Silva ES, et al. Physical activity and Incedent depression: a meta-analysis of prospective cohort studies. Am J Psychiatry. 2018;7:631-48.

15. Pogosova GV. Depression-a novel risk factor of ischemic heart disease and predictor of coronary death. Kardiologiia. 2002;42:86-90.

16. Pelletier R, Bacon SL, Arsenault A, Dupuis J, Laurin C, Blais L, et al. Relative associations between depression and anxiety on adverse cardiovascular events: does a history of coronary artery disease matter? A prospective observational study. BMJ Open. 2015;5:e006582.

17. Rugulies R. Depression as a predictor for coronary heart disease. A review and meta-analysis. Am J Prev Med. 2002;23:51-61.

18. Paganini-Hill A. Lifestyle practices and cardiovascular disease mortality in the elderly: the leisure world cohort study. Cardiol Res Pract. 2011:983764.

19. Carroll AJ, Carnethon MR, Liu K, Jacobs DR, Colangelo LA, Stewart JC, et al. Interaction between smoking and depressive symptoms with subclinical heart disease in the coronary artery risk development in young adults (CARDIA) study. Health Psychol. 2017;36:101-11.
20. Petter M, Blanchard C, Kemp KA, Mazoff AS, Ferrier SN. Correlates of exercise among coronary heart disease patients: review, implications and future directions. Eur J Cardiovasc Prev Rehabil. 2009;16:515-26.

21. Moller-Leimkuhler AM. Gender differences in cardiovascular disease and comorbid depression. Dialogues Clin Neurosci. 2007;9:71-83.

22. Blanchard C, Arthur HM, Gunn E. Self-efficacy and outcome expectations in cardiac rehabilitation: associations with women's physical activity. Rehabil Psychol. 2015;60:59-66.

23. Mosca L, Banka CL, Benjamin EJ, Berra K, Bushnell C, Dolor RJ, et al. Evidence-based guidelines for cardiovascular disease prevention in women: 2007 update. J Am Coll Cardiol. 2007:49:1230-50.

24. Trost SG, Owen N, Bauman AE, Sallis JF, Brown W. Correlates of adults' participation in physical activity: review and update. Med Sci Sports Exerc. 2002;34:1996-2001.

25. Bauman AE, Reis RS, Sallis JF, Wells JC, Loos RJ, Martin BW. Correlates of physical activity: why are some people physically active and others not? Lancet. 2012;380:258-71.

26. Thombs BD, Bass EB, Ford DE, Stewart KJ, Tsilidis KK, Patel U, et al. Prevalence of depression in survivors of acute myocardial infarction. J Gen Intern Med. 2006;21:30-8.

27. Meijer A, Conradi HJ, Bos EH, Thombs BD, van Melle JP, de Jonge P. Prognostic association of depression following myocardial infarction with mortality and cardiovascular events: a meta-analysis of 25 years of research. Gen Hosp Psychiatry. 2011;33:203-16.

28. van Lieshout J, Huntink E, Koetsenruijter J, Wensing M. Tailored implementation of cardiovascular risk management in general practice: a cluster randomized trial. Implement Sci. 2016;11:115.

29. Wensing M. The tailored implementation in chronic diseases (TICD) project: introduction and main findings. Implement Sci. 2017;12:5.

30. Huntink E, Heijmans N, Wensing M, van Lieshout J. Effectiveness of a tailored intervention to improve cardiovascular risk management in primary care: study protocol for a randomised controlled trial. Trials. 2013;14:433.

31. STROBE initiative group Strobe-statement. 2009. https://www.strobestatement.org/index.php?id=strobe-home. Accessed 10 Jan 2018.

32. Topolski TD, LoGerfo J, Patrick DL, Williams B, Walwick J, Patrick MB. The rapid assessment of physical activity (RAPA) among older adults. Prev Chronic Dis. 2006:3:A118.

33. Kroenke K, Spitzer RL, Williams JB. The PHQ-9: validity of a brief depression severity measure. J Gen Intern Med. 2001;16:606-13.

34. Hibbard JH, Stockard J, Mahoney ER, Tusler M. Development of the patient activation measure (PAM): conceptualizing and measuring activation in patients and consumers. Health Serv Res. 2004:35:1005-26.36

35. Mayer CJ, Steinman L, Williams B, Topolski TD, LoGerfo J. Developing a telephone assessment of physical activity (TAPA) questionnaire for older adults. Prev Chronic Dis. 2008;5:A24.

36. Yaroch AL, Nebeling L, Thompson FE, Hurley TG, Hebert JR, Toobert DJ, et al. Baseline design elements and sample characteristics for seven sites participating in the nutrition working Group of the Behavior Change Consortium. J Nutr. 2008;138:185S-92S.

37. Lani J. Statistics solutions. 2018. http://www.statisticssolutions.com/. Accessed 10 Jan 2018.

38. Vancampfort D, Stubbs B, Veronese N, Mugisha N, Koyanagi A. Correlates of physical activity among depressed older people in six low-income and middle income countries: Acommunity-bases cross-sectional study. Int J Geriatr Psychiatry. 2018;2:314-22.

39. Vancampfort D, Stubbs B, Sienaert P, Wyckaert S, Hert D, Rosenbaum S, Probst M. What are the factors that influence physical activity participation in individuals with depression? A review of physical activity correlater from 59 studies. Psychiatr Danub. 2015:3:210-24.

40. Blanchard CM, Reid RD, Morrin LI, McDonnell L, McGannon K, Rhodes RE, et al. Demographic and clinical determinants of moderate to vigorous physical activity during home-based cardiac rehabilitation: the home-based determinants of exercise (HOME) study. J Cardiopulm Rehabil Prev. 2010;30: 240-5.

41. Sallis JF, Prochaska JJ, Taylor WC. A review of correlates of physical activity of children and adolescents. Med Sci Sports Exerc. 2000;32:963-75.

42. Gaalema DE, Savage PD, Rengo JL, Cutler AY, Elliott RJ, Priest JS, et al. Patient characteristics predictive of cardiac rehabilitation adherence. J Cardiopulm Rehabil Prev. 2017;37:103-10. 